Article

\title{
Fabrication and catalytic behavior of hierarchically-structured nylon 6 nanofiber membrane decorated with silver nanoparticles
}

\author{
Huihui Zhao a, Weimin Kang a,b,*, Xiaomin Ma a, Nanping Deng a, Zongjie Li a, Bowen Cheng a,b,\# \\ a College of Textile, Tianjin Polytechnic University, Tianjin 300387, China \\ b State Key Laboratory of Separation Membranes and Membrane Processes, Tianjin Polytechnic University, Tianjin 300387, China
}

\section{A R T I C L E I N F}

\section{Article history:}

Received 5 August 2016

Accepted 29 August 2016

Published 5 January 2017

\section{Keywords:}

Electrospinning

Hierarchical structure

Ag nanoparticle

PA6 nanofiber membrane

Catalysis

\begin{abstract}
A B S T R A C T
A hierarchically-structured nylon 6 (PA6) nanofiber membrane decorated with silver nanoparticles (Ag NPs) was fabricated by electrospinning and impregnation methods. The as-fabricated hierarchically-structured Ag/PA6 nanofiber membrane (HS-Ag/PA6 NM) exhibits a morphology in which Ag NPs are deposited on the surfaces of both thick fibers and thin fibers. The content and size of the Ag NPs can be controlled by varying the concentration of the silver colloid solution. Compared with the non-hierarchically-structured Ag/PA6 nanofiber membrane, HS-Ag/PA6 NM has a higher specific surface area and exhibits a higher degradation rate for methylene blue of $81.8 \%-98.1 \%$ within 2 h. HS-Ag/PA6 NM can be easily recycled and exhibits good reusability. It retains a degradation rate for methylene blue of $83.5 \%$ after five consecutive cycles. The hierarchically-structured nanofiber membrane is therefore a potential nanocatalyst.
\end{abstract}

(C) 2016, Dalian Institute of Chemical Physics, Chinese Academy of Sciences. Published by Elsevier B.V. All rights reserved.

\section{Introduction}

The rapid development of economies and technology has led to increased attention to wastewater treatment, especially to effluent containing dyes and their decomposition products produced in the textiles industry [1]. Silver is a group IB transition metal with a valence shell electronic structure of $4 d^{10} 5 s^{1}$. Silver-based materials are widely applied in catalysis because of their specific physical and chemical structures [2].

Silver nanoparticles (Ag NPs) are often loaded on a support material such as microspheres [3], beads [4], films [5], and fibers [6], which limits their agglomeration and improves their catalytic activity. In situ reduction [7] and post-processing [8] are two common methods for loading Ag NPs onto supports. In situ reduction is simple and convenient, but most Ag NPs are distributed within the support, with only a few exposed on the surface, which is therefore an inefficient use of Ag NPs. In contrast, decorating Ag NPs on the surface of fiber supports through post-processing can yield silver-based materials with high catalytic activities [9].

Nanofibers are fibers with diameters of less than $100 \mathrm{~nm}$. The nanometer scale of the nanofibers makes them an efficient catalytic support [10]. Electrospinning is a versatile, effective, and widespread method for manufacturing long continuous nanofibers [11]. There are many reports of electrospun nanofiber membranes being used as catalytic supports for Ag NPs. Jang et al. [12] fabricated cellulose-based nanofibers by electrospinning and subsequent ultraviolet irradiation. The result-

\footnotetext{
* Corresponding author. Tel: +86-13920486699; E-mail: kweimin@126.com

\# Corresponding author. Tel: +86-13802163663; E-mail: bowen15@tjpu.edu.cn

This work was supported by the National Natural Science Foundation of China (51673148), National Basic Research Program of China (2014CB660813), National Key Technology Support Program (2015BAE01B03), Innovation Fund for Technology of China (14C26211200298), and Innovation Fund for Technology of Tianjin (14TXGCCX00014,14ZXCXGX00776).

DOI: 10.1016/S1872-2067(16)62545-7 | http://www.sciencedirect.com/science/journal/18722067 | Chin. J. Catal., Vol. 38, No. 1, January 2017
} 
ing cellulose nanofiber matrices were loaded with $\mathrm{Ag}^{+}$or $\mathrm{Ag}$ NPs, and demonstrated potential as catalytic membranes for the sensing of specific chemicals. Liu et al. [13] fabricated $\mathrm{Ag} / \mathrm{PS}$ nanofibers by a combination of electrospinning and in situ reduction. The resulting membranes exhibited excellent catalytic activity for the degradation of dye pollutants. Gao et al. [14] fabricated Ag/PAN fiber networks, which exhibited activity for the degradation of 4-NP under general conditions.

Recent advances in electrospinning technology have allowed the fabrication of specifically-structured nanofiber membranes, such as core-shell structures [15], hollow structures [16], porous structures [17], and web-like structures $[18,19]$. Hierarchically-structured nanofibers with multiscale organizations have been inspired by the hierarchical structures of trees consisting of many trunks and branches. Such nanofibers have exhibited high levels of functionality and performance. This has been because of the high surface area provided by their thin fibers, and excellent mechanical properties provided by their thick fibers [20-22]. Specifically-structured nanofiber membranes have been applied in filters [23], sensors [24], and catalysts [25]. Bai et al. [26] fabricated tree-like hierarchically-structured $\mathrm{TiO}_{2}$ nanofiber (NF)/ZnO nanorod (NR) materials by the electrospinning of $\mathrm{TiO}_{2} \mathrm{NFs}$ and hydrothermal growth of ZnO NRs. The resulting structures yielded high photocatalytic activity because of their high specific surface area, high rate of mass transfer, and thus readily accessible reaction sites. Shi et al. [25] fabricated branch-like carboxylated multiwalled carbon nanotube/chitosan nanofibrous membranes by simultaneous electrospinning and spraying. The resulting membranes exhibited improved rejection of $161 \%-166 \%$ and 80\%-90\% toward methylene blue (MB) and methyl orange, respectively. We recently fabricated hierarchically-structured nanofiber membranes by adding tetrabutylammonium chloride (TBAC) into PVDF/dimethyl formamide/acetone solution, via one-step electrospinning. The resulting tree-like PVDF nanofiber membranes exhibited high mechanical strength and high specific surface areas [27].

Nylon 6 (PA6) is a widely used polymer, which is low cost, exhibits strong chemical and thermal stability, and is hydrophilic $[28,29]$. The molecular chains of PA6 can coordinate with Ag, making PA6 a potential Ag NP support [30-32]. In the current study, a hierarchically-structured Ag/PA6 nanofiber membrane (HS-Ag/PA6 NM) was fabricated by adding TBAC into PA6/HCOOH solution, via one-step electrospinning and silver colloid solution impregnation methods. The resulting HS-Ag/PA6 NM exhibits excellent catalytic activity in the reduction of MB. This enhanced catalytic performance results from the high specific surface area of HS-Ag/PA6 NM. Because the membrane is easily recovered and reused, it has potential practical use in catalysis.

\section{Experimental}

\subsection{Materials}

Nylon 6 granules (PA6, $M_{w}=16000$ ) were purchased from Ube Industries Ltd. PVP was purchased from Jiaozuo Meida
Fine Chemical Co., Ltd. Formic acid ( $\mathrm{HCOOH})$ and silver nitrate $\left(\mathrm{AgNO}_{3}\right)$ were purchased from Tianjin Yingda Rare Chemical Reagents Factory. TBAC was purchased from Tianjin Guangfu Chemical Reagent Co. $\mathrm{NaBH}_{4}$ and $\mathrm{NaOH}$ were purchased from Tianjin Fengchuan Chemical Reagent Technologies Co. MB and acid magenta were purchased from Tianjin Tensing Fine Chemical Research Develop Center. All reagents except PA6 and PVP were of analytical grade, and were used without further purification. Deionized water was used through all experiments.

\subsection{Preparation of nanofiber membranes}

PA6/TBAC solution was prepared by mixing 14 wt.\% PA6 and $4 \mathrm{wt} . \%$ TBAC with $\mathrm{HCOOH}$ and stirring for $10-12 \mathrm{~h}$ to form a stable and homogeneous solution. For comparison, a solution without TBAC was also prepared. These solutions were used to prepare nanofiber membranes by electrospinning with an applied voltage of $45 \mathrm{kV}$, distance from collector to syringe tip of $15 \mathrm{~cm}$, and injection rate of $0.1 \mathrm{~mL} / \mathrm{h}$. The inner diameter of the syringe was $0.45 \mathrm{~mm}$. The temperature was kept at $25 \pm 2{ }^{\circ} \mathrm{C}$, and the relative humidity was $(35 \pm 2) \%$. The nanofiber membranes were collected on surface of a grounded aluminum foil. The membranes were then washed with distilled water, dried at $60{ }^{\circ} \mathrm{C}$, and cut into $2 \mathrm{~cm} \times 2 \mathrm{~cm}$ pieces for further use. Membranes fabricated with and without the addition of TBAC were denoted HS-PA6 NM and PA6 NM, respectively.

\subsection{Preparation of Ag-decorated PA6 nanofiber membranes}

PVP $(0.0844 \mathrm{~g})$ was added to $60 \mathrm{~mL}$ of deionized water and stirred for $20 \mathrm{~min} . \mathrm{NaBH}_{4}$ solution $(0.008 \mathrm{~mol} / \mathrm{L}, 40 \mathrm{~mL})$ and 10 $\mathrm{mL}$ of $\mathrm{AgNO}_{3}$ solution $(0.006 \mathrm{~mol} / \mathrm{L})$ were prepared under dark conditions. The PVP and $\mathrm{NaBH}_{4}$ solutions were then mixed thoroughly, and the $\mathrm{AgNO}_{3}$ solution was then added slowly, yielding a silver colloid solution named solution A. Similar solutions were prepared with concentrations of $0.012 \mathrm{~mol} / \mathrm{L} \mathrm{Ag-}$

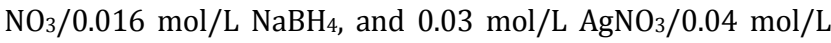
$\mathrm{NaBH}_{4}$, and were named solution $\mathrm{B}$ and solution $\mathrm{C}$, respectively. $\mathrm{Ag} / \mathrm{PA} 6 \mathrm{NM}$ and HS-Ag/PA6 NM were fabricated by the impregnation method, and $0.1 \mathrm{~g}$ of nanofiber membranes were immersed in $30 \mathrm{~mL}$ of silver colloid solution for $5 \mathrm{~h}$ in the dark. The membranes were then washed with deionized water for three times, and dried at $60^{\circ} \mathrm{C}$. PA6 NM immersed in solutions $A, B$, and $C$ were named samples 1,2 , and 3 , respectively. HS-PA6 NM immersed in solutions $A, B$, and $C$ were named samples 4, 5, and 6, respectively. The fabrication of HS-Ag/PA6 NM is shown schematically in Fig. 1.

\subsection{Characterization}

The morphologies of the electrospun membranes were observed using field-emission scanning electron microscopy (FE-SEM, S-4800, Hitachi, Japan). Energy dispersive X-ray spectroscopy (EDS) observations were also carried out using the FE-SEM instrument. The size, shape, and deposition of the Ag NPs on the fiber surface were investigated using transmis- 


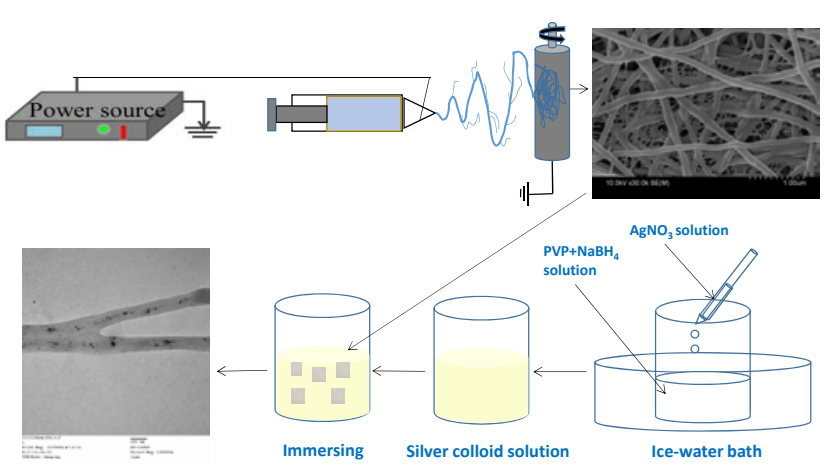

Fig. 1. The schematic illustration for the fabrication of HS-Ag/PA6NM.

sion electron microscopy (TEM, H-7650, Hitachi, Japan). X-ray photoelectron spectroscopy (XPS) was carried out with K-alpha $\mathrm{X}$ radiation using a Thermo Fisher Co. instrument (England). The specific surface areas of PA6 NM and HS-PA6 NM were investigated by nitrogen adsorption measurements (Quantachrome Instruments Autosorb-iO, USA).

\subsection{Catalytic tests}

The catalytic activities of the samples were investigated by their degradation of MB. MB is a standard model contaminant for catalysis tests. Before degradation, $0.1 \mathrm{~g}$ of the nanofiber membranes was dispersed in $50 \mathrm{~mL}$ MB solutions with concentrations of 10 and $20 \mathrm{mg} / \mathrm{L}$ for $2 \mathrm{~h}$ in the dark, to ensure adsorption equilibrium. A certain amount of $\mathrm{NaBH}_{4}$ was then added, and $0.1 \mathrm{~g}$ of $\mathrm{NaOH}$ was added to stabilize the $\mathrm{NaBH}_{4}$. At different time intervals, 1-2 $\mathrm{mL}$ of test solution was removed. The absorbance of this aliquot at the wavelength of maximum absorption intensity of MB $\left(\lambda_{\max }=665 \mathrm{~nm}\right)$ was quickly measured using a UV-2401 Shimadzu spectrophotometer. The degradation rate of $\mathrm{MB}$ was expressed as $D=\left(1-\left(C / C_{0}\right)\right) \times 100 \%$, where $C_{0}$ and $C$ are the initial and residual concentrations of $\mathrm{MB}$, respectively.
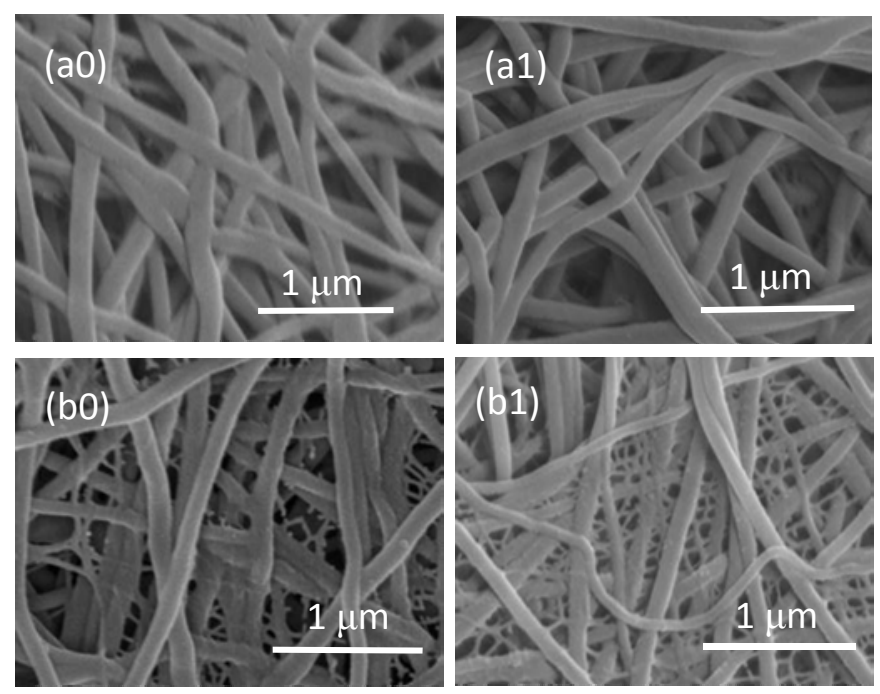

\section{Results and discussion}

\subsection{Morphology of HS-Ag/PA6 NM}

Fig. 2 shows FE-SEM images of PA6 NM and HS-PA6 NM before and after immersing in silver colloid solution. The nanofibers formed in the absence of TBAC exhibit a common circular structure (Fig. 2(a1)). The nanofibers formed in the presence of TBAC exhibit a hierarchically structure (Fig. 2(b1)) composed of thick and thin fibers. The formation of the hierarchically structure can be explained by the presence of TBAC. TBAC is an organic branched salt [33], which increases the electrical conductivity of the solution, and destabilizes the electrospinning jet. Splitting of the jet occurs when the excess charge density is above a certain threshold, at which point the electric forces overcome the surface tension. The presence of TBAC also decreases the forces between PA6 molecules because of its steric bulk, which promotes slipping of the jet. The formation mechanism was reported in our previous study [27]. Splitting of the jet also leads to a change in the nanofiber diameter, since the diameter of HS-PA6 NM is smaller than that of PA6 NM. The nanofiber diameter of PA6 NM is $80-140 \mathrm{~nm}$, as shown in Fig. 3(a1). The diameters of the thick and thin nanofibers of HS-PA6 NM are $50-120 \mathrm{~nm}$ and $10-50 \mathrm{~nm}$, respectively, as shown in Figs. 3(a2) and 3(a3), respectively. Immersing in the silver colloid solution and water washing has no obvious effect on the morphologies of the nanofibers.

EDS analysis of the as-fabricated nanofiber membranes is shown in Table 1. The higher recorded Ag content of HS-PA6 NM demonstrates that the Ag content on the surface of HS-PA6 NM is higher than that on the surface of PA6 NM, probably because of the high specific surface area of HS-PA6 NM. The Ag content increases with increasing concentration of the silver colloid solution, as shown in Table 1. Therefore, the content of Ag NPs loaded on the fibers could be controlled by the concentration of the silver colloid solution.

TEM images of PA6 NM and HS-PA6 NM nanofibers deco-

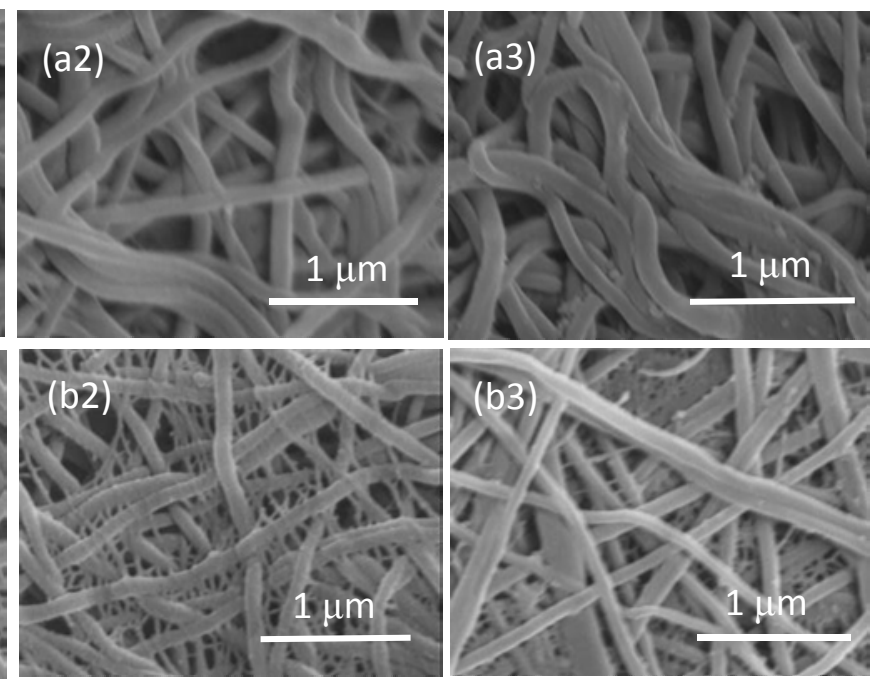

Fig. 2. FE-SEM images of nanofiber membranes. (a0) PA6 NM; (a1) Sample 1; (a2) Sample 2; (a3) Sample 3; (b0) HS-PA6 NM; (b1) Sample 4; (b2) Sample 5; (b3) Sample 6. 

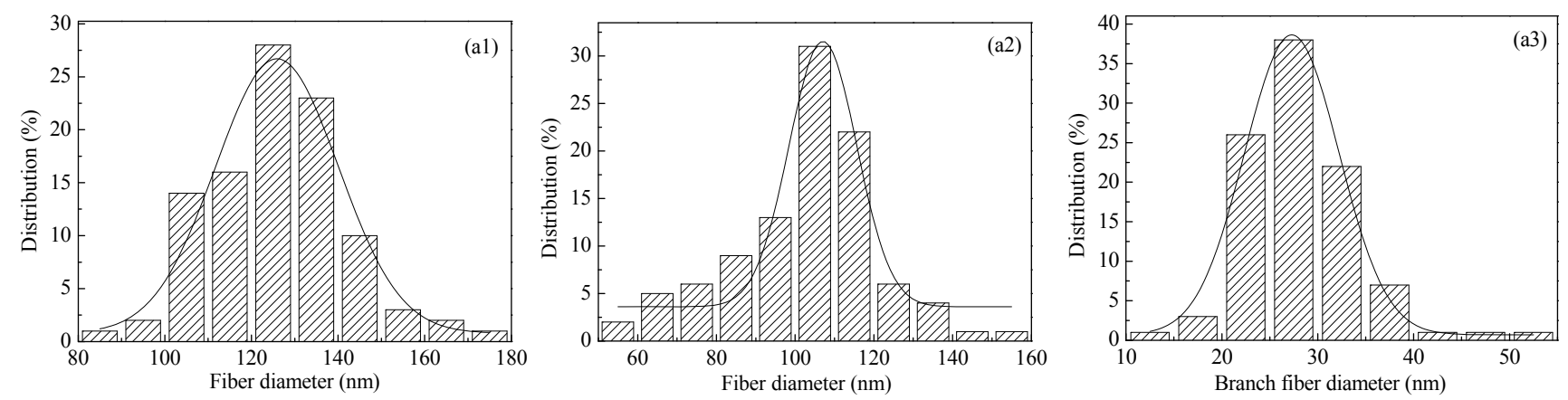

Fig. 3. Fiber diameter distribution of different nanofiber membranes. (a1) PA6 NM; (a2) Thick fibers of HS-PA6 NM; (a3) Thin fibers of HS-PA6 NM.

rated with Ag NPs are shown in Fig. 4. The size of the Ag NPs could also be controlled by the concentration of the silver colloid solution. The mean sizes of the Ag NPs in samples 1, 2, 3, 4, 5 , and 6 are estimated to be approximately 11.2, 27.4, 50.1, 8.6, 21.3, and $39.2 \mathrm{~nm}$, respectively (Fig. 5). This indicates that the size of the Ag NPs increases with increasing silver colloid solution concentration. The Ag NPs distributed on PA6 NM are

\section{Table 1}

The content of element in different nanofiber membranes analyzed by EDS.

\begin{tabular}{lccccc}
\hline \multirow{2}{*}{ Sample } & \multicolumn{5}{c}{ Element content (at\%) } \\
\cline { 2 - 6 } & $\mathrm{C}$ & $\mathrm{N}$ & $\mathrm{O}$ & $\mathrm{Cl}$ & $\mathrm{Ag}$ \\
\hline PA6 NM & 69.62 & 16.87 & 12.12 & 0.03 & 0 \\
Sample 1 & 74.99 & 18.30 & 11.63 & 0.06 & 0.41 \\
Sample 2 & 69.02 & 18.17 & 11.23 & 0.05 & 1.28 \\
Sample 3 & 67.70 & 16.75 & 11.29 & 0.11 & 4.15 \\
HS-PA6 NM & 73.86 & 14.07 & 7.30 & 4.95 & 0 \\
Sample 4 & 70.22 & 16.86 & 10.11 & 1.32 & 1.50 \\
Sample 5 & 63.73 & 18.43 & 12.12 & 1.02 & 4.80 \\
Sample 6 & 46.8 & 20.19 & 21.56 & 4.68 & 6.77 \\
\hline
\end{tabular}
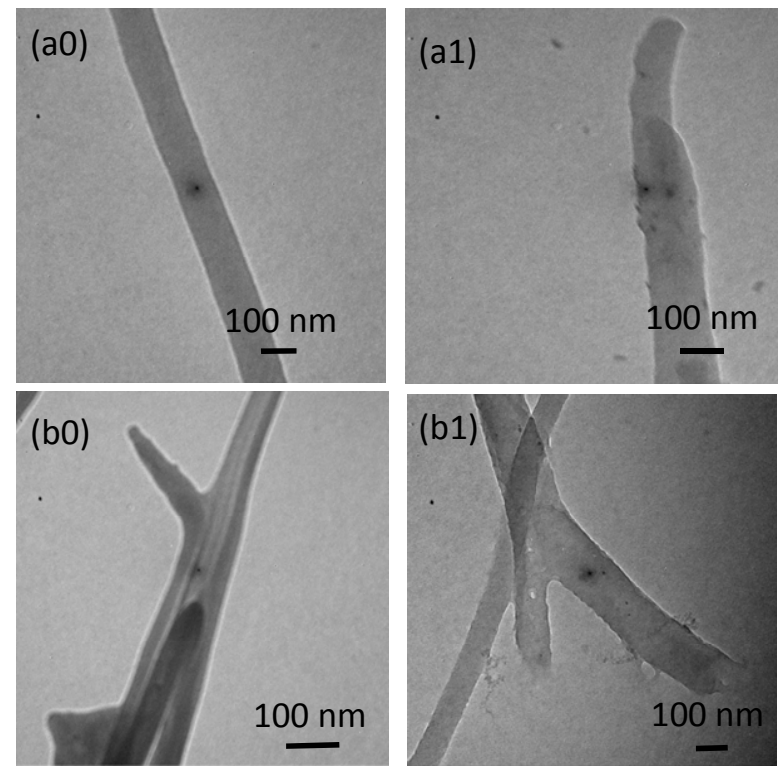

Fig. 4. TEM images and color of different nanofiber membranes. (a0) PA6 NM; (a1) Sample 1; (a2) Sample 2; (a3) Sample 3; (b0) HS-PA6 NM; (b1) Sample 4; (b2) Sample 5; (b3) Sample 6.
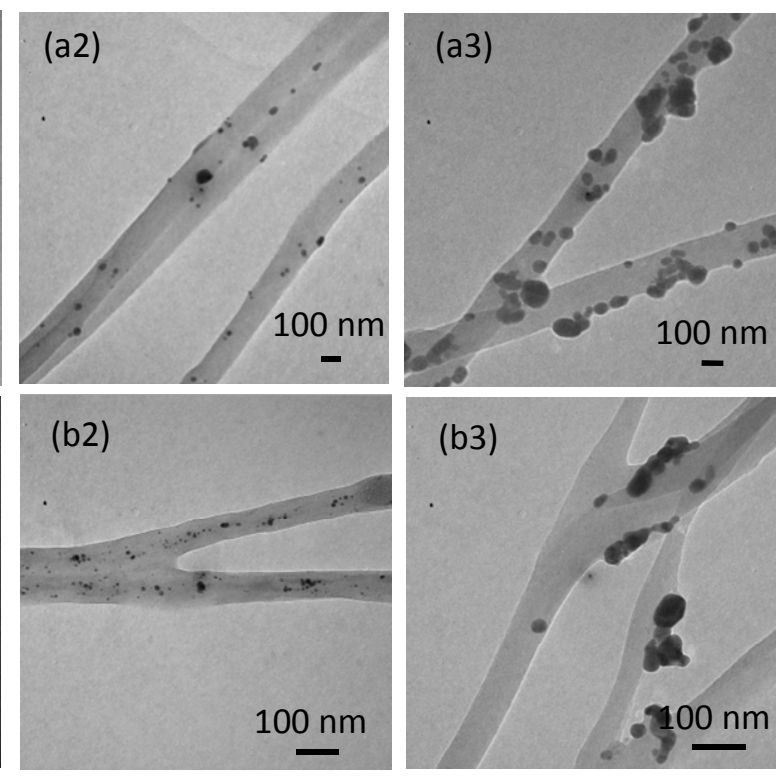

slightly larger than those on HS-PA6 NM, when immersed in the same concentration silver colloid solution. This further indicates that HS-PA6 NM is better able to disperse the Ag NPs, compared to PA6 NM. The size and content of Ag NPs reportedly have a pronounced effect on the color of the silver colloid solution [34]. In the current study, the colors of samples varied from pale yellow to light black with increasing Ag colloid size and concentration, as shown in Fig. 4.

\subsection{Structure of HS-Ag/PA6 NM}

BET analysis was also used to investigate the specific surface areas of PA6 NM and HS-PA6 NM. Fig. 6 shows nitrogen adsorption-desorption isotherms for PA6 NM and HS-PA6 NM. The isotherms of both membranes could be categorized as type IV from BDDT, indicating the presence of mesopores in the nanofiber membranes [35]. The BET specific surface area of HS-PA6 NM is $21.867 \mathrm{~m}^{2} / \mathrm{g}$, which is higher than that of PA6 NM $\left(15.826 \mathrm{~m}^{2} / \mathrm{g}\right)$. The higher specific surface area facilitates a higher Ag NP loading, in accordance with the EDS results in Table 1. 

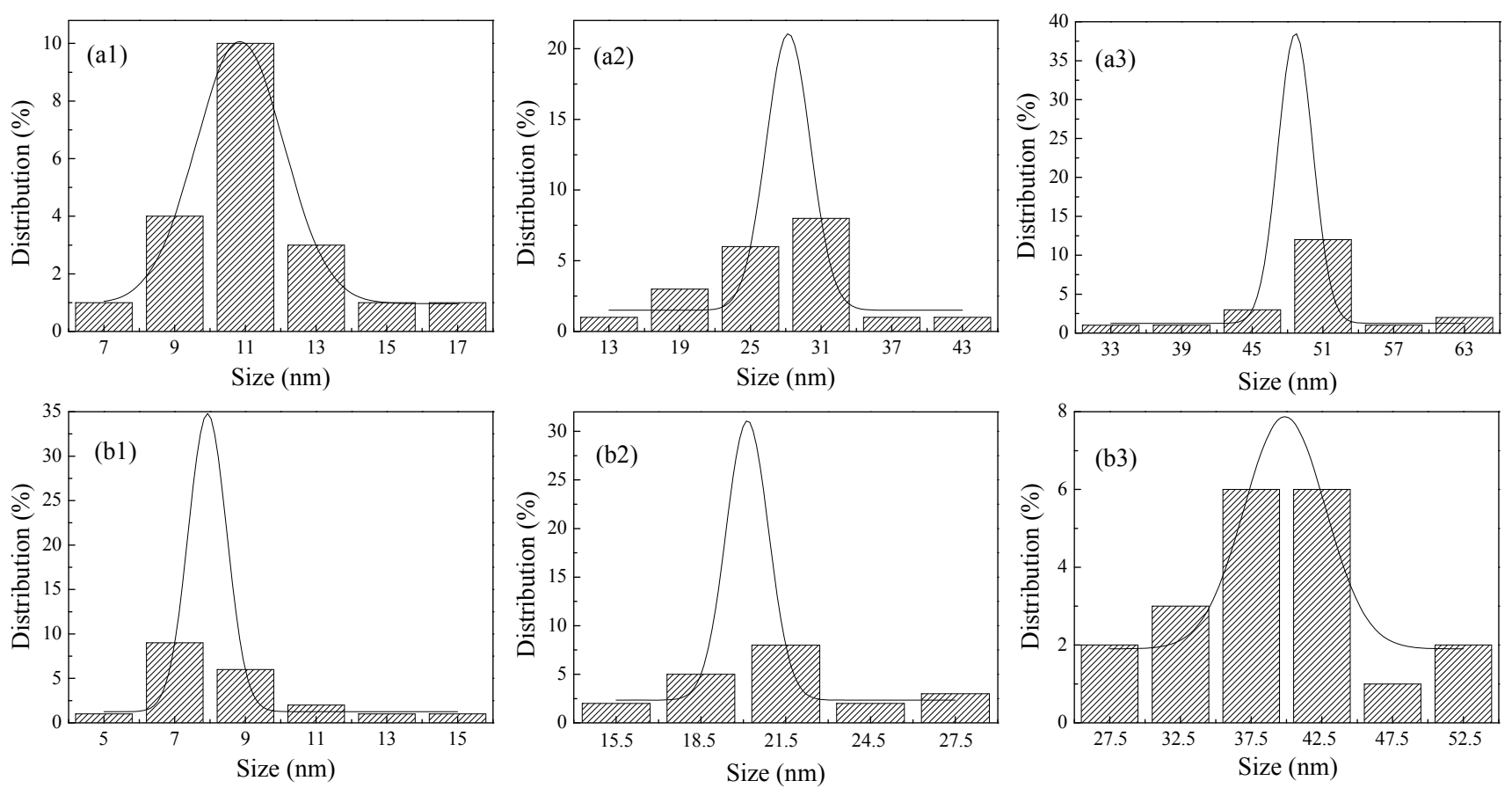

Fig. 5. The size distribution of Ag Nps of various samples. (a1) Sample 1; (a2) Sample 2; (a3) Sample 3; (b1) Sample 4; (b2) Sample 5; (b3) Sample 6.

Fig. 7 shows the XPS spectrum of HS-Ag/PA6 NM. C, N and O exist throughout the whole region, as shown in Fig. 7(a). High-resolution spectra of the Ag $3 d$ region are shown in Fig. 7 (b), (c), and (d). The spectra of samples 4, 5, and 6 contain two peaks at binding energies of about 368 and $374 \mathrm{eV}$. Curve fitting indicates that two peaks centered at 368.2 and $374.2 \mathrm{eV}$ are attributed to metallic $\mathrm{Ag}^{0}$. Another two peaks centered at 368.8 and $374.8 \mathrm{eV}$ are attributed to non-reduced silver $\left(\mathrm{Ag}^{+}\right)$ trapped in the fiber membrane [36]. The $\mathrm{Ag}^{0}$ contents of samples 5 and 6 are higher than that in sample 4. This is because the low $\mathrm{NaBH}_{4}$ concentration in solution A results in the incomplete reduction of $\mathrm{AgNO}_{3}$. High-resolution XPS spectra of the $\mathrm{O}$ and $\mathrm{N}$ regions were investigated to confirm the presence of coordination bonds between PA6 molecular chains and Ag. Fig. 7(e) shows a peak associated with oxygen doubly bound to carbon. This indicates that the 0 predominantly exists in the

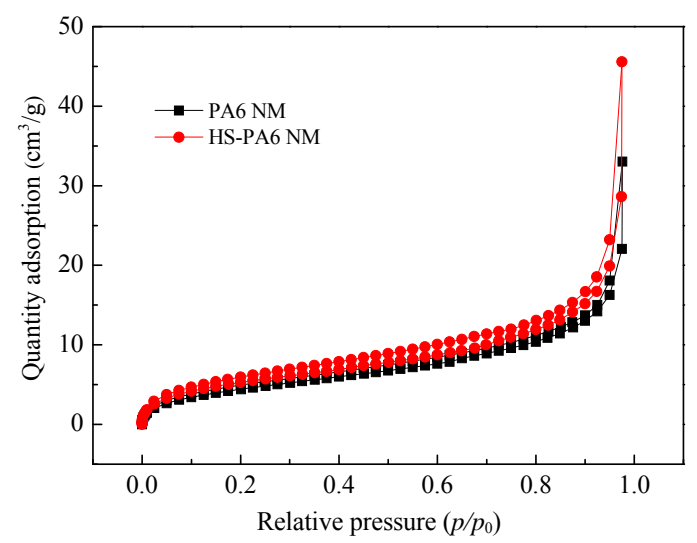

Fig. 6. Nitrogen adsorption-desorption isotherms of PA6 NM and HS-PA6 NM. form of carbonyl $(\mathrm{C}=0)$ groups in PA6. The $01 s$ peak of the carbonyl oxygen atom in HS-Ag/PA6 NM shifts to higher binding energy (532.1 eV) compared with HS-PA6 NM (531.6 eV). This suggests a decrease in electron density because of coordination between PA6 and Ag, in which electrons transfer from the carbonyl oxygen atom to Ag. The peak observed at $532.9 \mathrm{eV}$ arises due to the existence of $\mathrm{NO}^{3-}$. Fig. 7(e) shows a distinctive peak at $400.44 \mathrm{eV}$, which is attributed to the nitrogen atoms of PA6. The $\mathrm{N} 1 \mathrm{~s}$ peak does not appear to be influenced by the presence of Ag, since no obvious shift is seen in Fig. 7(f). The new peak at $406.8 \mathrm{eV}$ could also be attributed to the existence of $\mathrm{NO}^{3-}$. These result reveal the presence of coordination bonds between the carbonyl oxygen atom and Ag, which would inhibit the agglomeration of Ag NPs and contribute to their immobilization [30].

\subsection{Catalytic properties of HS-Ag/PA6 NM}

The catalytic degradation capacity of HS-Ag/PA6 NM was investigated by the reduction of $\mathrm{MB}$ using the reducing agent $\mathrm{NaBH}_{4}$. MB has a characteristic absorption peak at $665 \mathrm{~nm}$ [37], the intensity of which is proportional to its concentration in solution. A decrease in absorption can be attributed to the degradation of MB [38]. The rate of degradation can be determined by measuring the intensity of the absorbance of the MB solution at $665 \mathrm{~nm}$ at different time intervals. Fig. 8 shows the corresponding ultraviolet-visible (UV-vis) absorption spectra and degradation rate of MB. The difference in the dye adsorption for the three samples is shown in Fig. 8(c). The adsorption activity of HS-Ag/PA6 NM is higher than those of Ag/PA6 NM and PA6 NM, and the adsorption activity of PA6 NM is slightly higher than that of Ag/PA6 NM. This can be explained by the wick- 

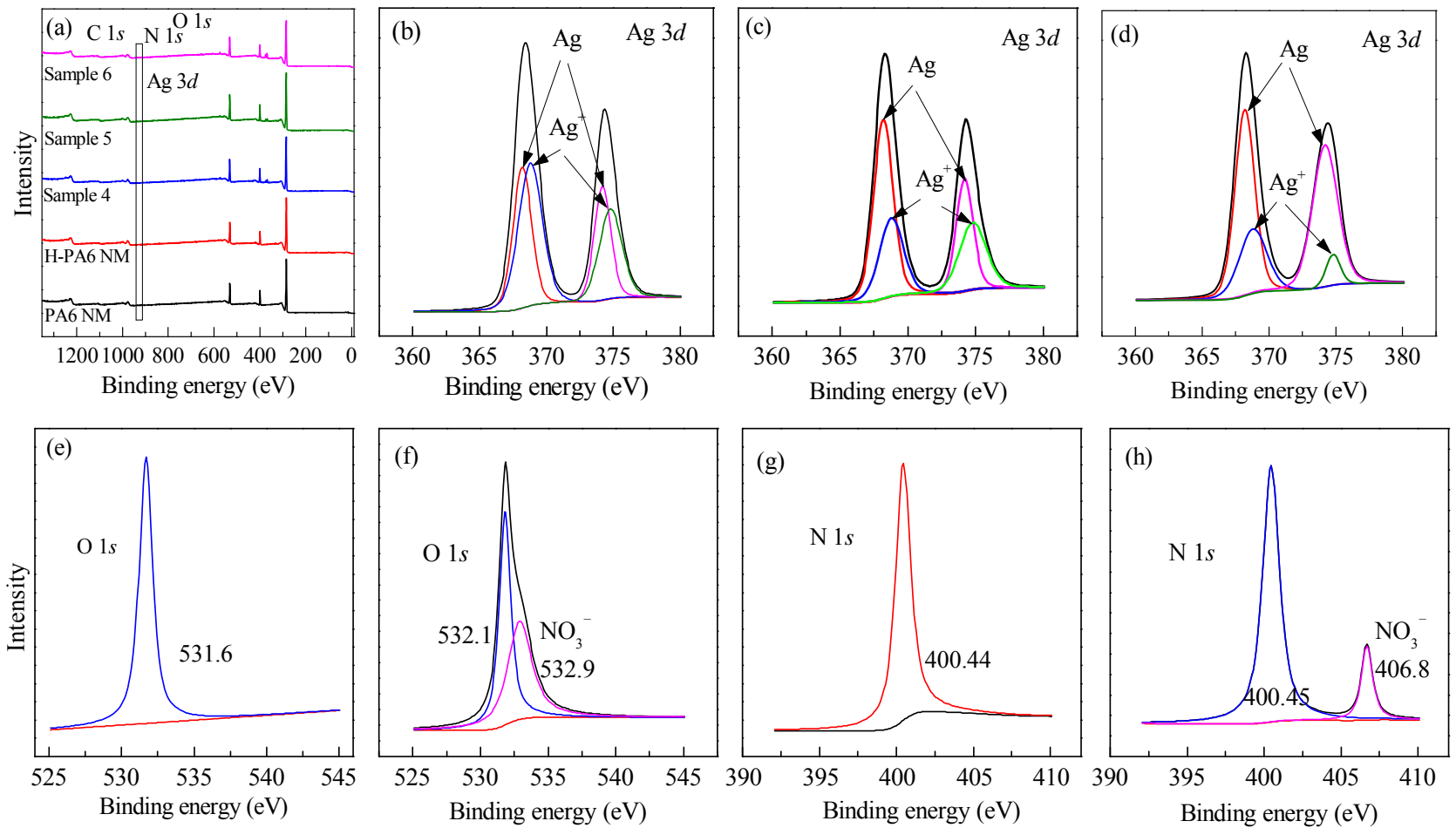

Fig. 7. XPS spectra of different nanofiber membranes. (a) Full spectra; (b) Ag $3 d$ spectrum of sample 4; (c) Ag $3 d$ spectrum of sample 5; (d) Ag 3d spectrum of sample 6; (e) $01 s$ spectrum of HS-PA6 NM; (f) $01 s$ spectrum of sample 5; (g) N $1 s$ spectrum of HS-PA6 NM; (h) N $1 s$ spectrum of sample 5.

ing progress. The presence of thinner fibers accelerates wicking. More MB molecules adsorb to the fibers, which promotes the degradation of MB. The absorbance at $665 \mathrm{~nm}$ gradually decreases with increasing reaction time, as the solution color changes from blue to colorless. The fastest degradation occurs in the catalytic system containing HS-Ag/PA6 NM, and which the MB color appears lighter than that in the system containing $\mathrm{Ag} / \mathrm{PA} 6 \mathrm{NMs}$, after a given time interval. This indicates that HS-Ag/PA6 NM has a better catalytic degradation capacity than $\mathrm{Ag} / \mathrm{PA} 6 \mathrm{NMs}$. MB degradation is almost complete after $2 \mathrm{~h}$ for the HS-Ag/PA6 NM system, as shown in Fig. 8(b). The degradation kinetics were then investigated. The linear region of the MB degradation with time can be accounted for using a pseudo first-order model, called the Langmuir-Hinshelwood (L-H) model [8]. Kinetic analysis was performed to understand the MB degradation procedure. Rate constants for MB degradation $(k)$ over the samples were calculated and are shown in Fig. $8(d)$. The rate constants in the presence of PA6 NM, Ag/PA6 $\mathrm{NM}$, and HS-Ag/PA6 NM are $-2.36 \times 10^{-3},-1.271 \times 10^{-2}$, and $-1.997 \times 10^{-2} \mathrm{~min}^{-1}$, respectively. HS-Ag/PA6 NM has the highest catalytic activity, as evidenced by its highest rate constant. The results demonstrate that the catalytic support plays an important role in the degradation of MB, and that HS-Ag/PA6 NM with its high specific surface area is a potential catalyst.

The catalytic degradation capacity of HS-Ag/PA6 NM immersed in silver colloid solutions of different concentrations was also investigated. Fig. 9 shows the MB degradation rate in the presence of the different nanofiber membranes. The adsorption activities follow the trend: sample $4>$ sample $5>$ sample 6 , but the differences are minor. Samples 4,5 , and 6 exhibit excellent catalytic properties. Their degradation rates indicate catalytic activities following the trend: sample 5 $(98.1 \%)>$ sample $4(92.5 \%)>$ sample $6(88.8 \%)$. The catalytic activity of HS-Ag/PA6 NM clearly depends on the Ag NP content and size. The obvious effects of the size and shape of $\mathrm{Ag}$ NPs on their antibacterial and catalytic properties have been previously reported [39]. The size of the Ag NPs in sample 4 is small $(8.6 \mathrm{~nm})$, but the Ag content is low (1.5\%). The Ag content in sample 6 is high (6.8\%), but the large size of the Ag NPs promotes aggregation (Table 1), which weakens the nanometer size effect. Sample 5 contains Ag NPs of suitable size $(21.3 \mathrm{~nm})$ and content (4.80\%), so exhibits the highest catalytic activity.

The effect of the initial $\mathrm{NaBH}_{4}$ concentration on the catalytic degradation of MB was investigated by varying the $\mathrm{NaBH}_{4}$ concentration from 0 to $0.5 \mathrm{~mol} / \mathrm{L}$. $0.1 \mathrm{~g}$ of HS-Ag/PA6 NM was immersed in $10 \mathrm{mg} / \mathrm{L} \mathrm{MB}$ solutions with different $\mathrm{NaBH}_{4}$ concentrations, and the resulting degradation rates were investigated. Fig. 10 shows that the MB degradation rate MB decreases with increasing $\mathrm{NaBH}_{4}$ concentration. Thus, a higher $\mathrm{NaBH}_{4}$ concentration promotes MB degradation, which can be explained by the catalytic mechanism. The catalytic reducing of MB by $\mathrm{NaBH}_{4}$ is shown in Fig. 11. Ag NPs on the fiber surface act as electron transfer pathways in the oxidation-reduction system. Nucleophilic $\mathrm{BH}^{4-}$ donates electrons to Ag NPs, while electrophilic MB receives electrons from Ag NPs [15]. Ag NPs accelerate the transfer of electrons, because of their electronic properties [40]. The reduction product of MB is Leucomethylene blue, as shown in the reaction equation. The $\mathrm{NaBH}_{4}$ con- 

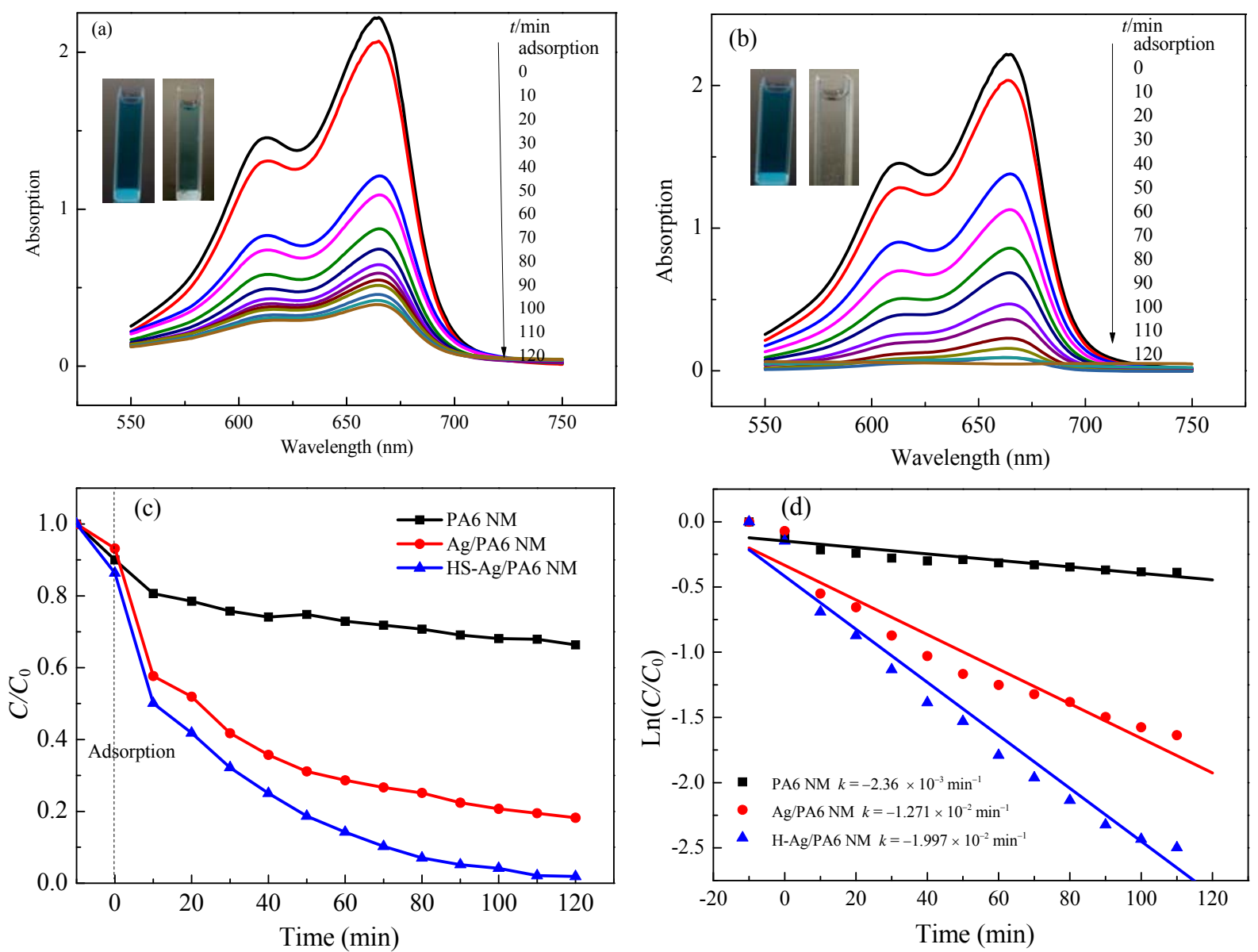

Fig. 8. The UV-visible absorption spectra and degradation $\left(C / C_{0}\right)$ of MB. (a) Ag/PA6 NM; (b) HS-Ag/PA6 NM; (c) Degradation rate; (d) Kinetics of MB degradation in $2 \mathrm{~h}$.

centration therefore has a significant effect on the degradation of MB.

The degradation rate for different initial MB concentrations was also investigated, and the results are shown in Fig. 12. Sample 5 exhibits a high adsorption activity for the MB solution $(10 \mathrm{mg} / \mathrm{L})$, with a degradation rate of $98.1 \%$. Its degradation rate for the MB solution $(20 \mathrm{mg} / \mathrm{L})$ decreases slightly to $84 \%$, perhaps because the $\mathrm{NaBH}_{4}$ concentration is insufficient to provide enough electrons for the reduction of MB. The stability

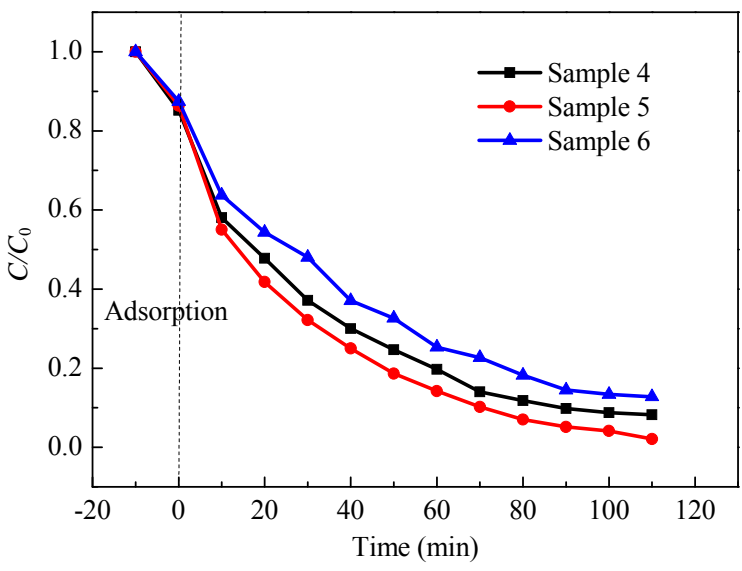

Fig. 9. Influence of silver colloid solution on MB degradation. of HS-Ag/PA6 NM was then investigated. Used catalyst was thoroughly washed with distilled water after each run, dried at $60{ }^{\circ} \mathrm{C}$, and then reused in the same procedure for up to five times. The degradation results are shown in Fig. 13. The catalytic activities of HS-Ag/PA6 NM after runs 1, 2, 3, 4, and 5 are $98.1 \%, 93 \%, 89 \%, 86 \%$, and $83.5 \%$, respectively. There is some decline in degradation rate after the five cycles, but HS-Ag/PA6 NM still maintains a satisfactory catalytic reduction capacity for MB.

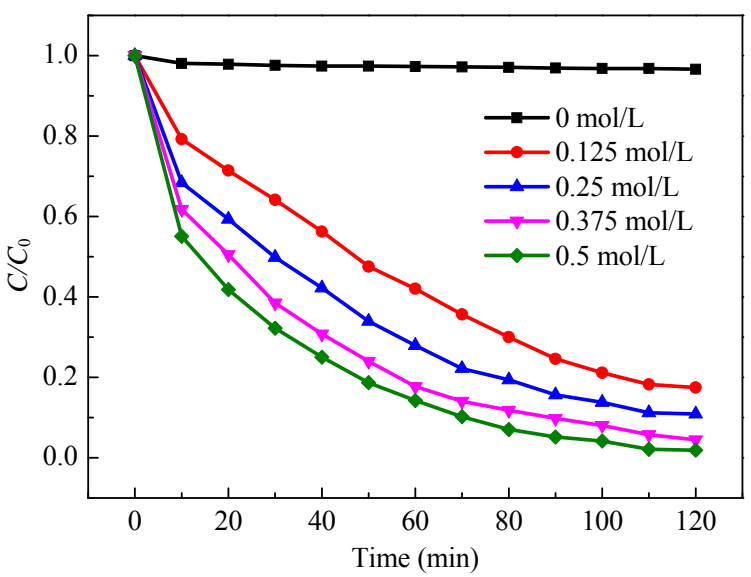

Fig. 10. Influence of $\mathrm{NaBH}_{4}$ concentration on $\mathrm{MB}$ degradation. 


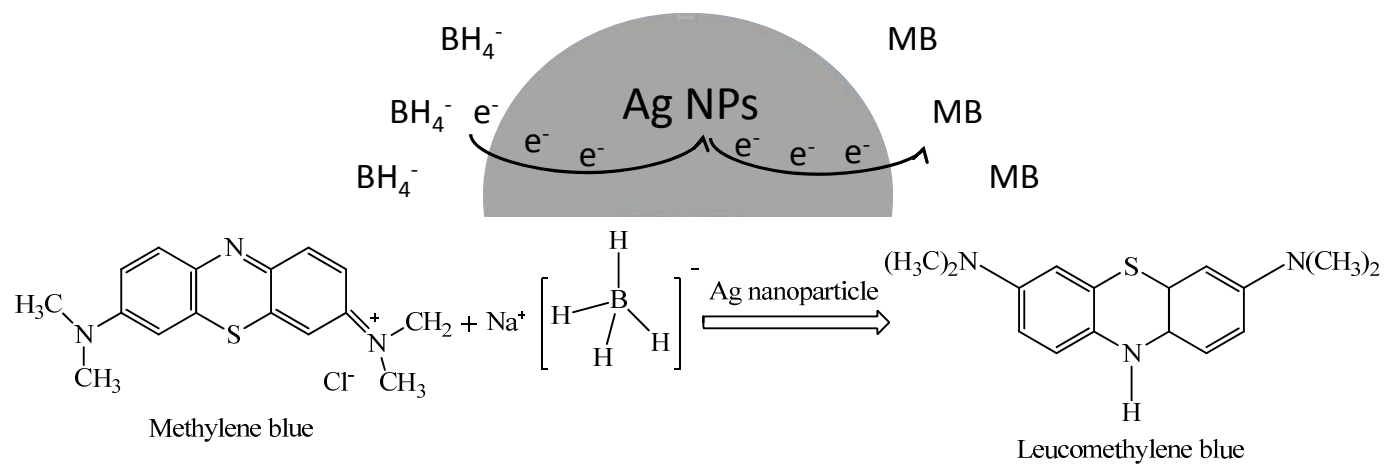

Fig. 11. The mechanism of catalytic electron transfer on the surface of nanofibers and the reaction equation.

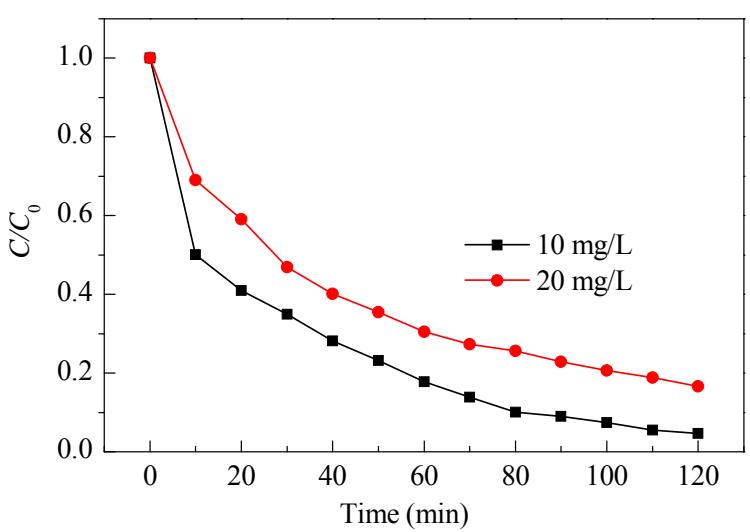

Fig. 12. Influence of initial concentration on degradation of MB.

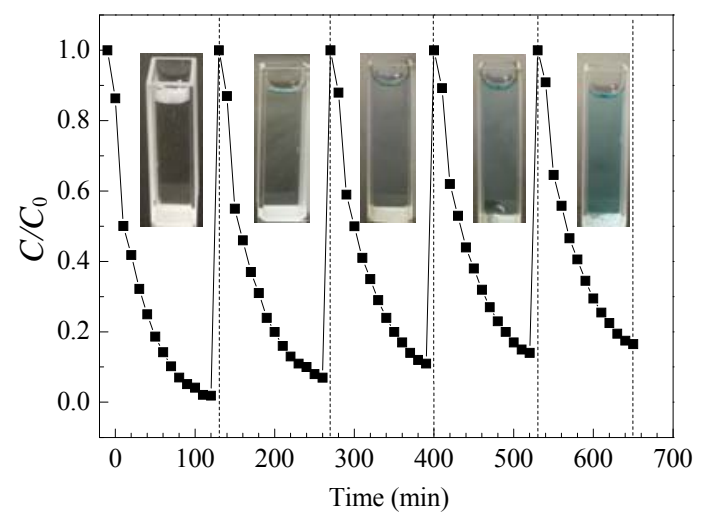

Fig. 13. Catalytic recycling of HS-Ag/PA6 NM.

\section{Conclusions}

HS-Ag/PA6 NM was fabricated and exhibited a high surface area and high accessibility of active sites. Coordination bonds form between the molecular chains of PA6 and Ag, which promote the immobilization of Ag NPs on the high surface area fibers. The content and size of the Ag NPs could be controlled by the concentration of the silver colloid solution. HS-Ag/PA6 NM exhibits excellent catalytic activity for the reduction of MB in the presence of $\mathrm{NaBH}_{4}$, because the fiber support provides readily accessible active sites. HS-Ag/PA6 NM can be readily recycled, and its $\mathrm{MB}$ degradation rate is $83.5 \%$ after five con- secutive cycles. These findings provide a potential platform for fabricating other noble metal nanocatalysts with readily accessible active sites.

\section{References}

[1] J. H. Weisburger, Mutat. Res.-Fundam. Mol. Mech. Mutagen., 2002, 506, 9-20.

[2] D. D. Lin, H. Wu, R. Zhang, W. Pan, Chem. Mater., 2009, 21, 3479-3484.

[3] Y. Liu, K. Zhang, X. S. Yin, W. Z. Wang, H. J. Zhu, J. Magn. Magn. Mater., 2016, 403, 18-29.

[4] E. Murugan, P. Shanmugam, Bull. Mater. Sci., 2015, 38, 629-637.

[5] I. M. Arabatzis, T. Stergiopoulos, M. C. Bernard, D. Labou, S. G. Neophytides, P. Falaras, Appl. Catal. B, 2003, 42, 187-201.

[6] P. V. Mayuri, P. Ramesh, J. Mater. Sci., 2016, 51, 2739-2746.

[7] M. Arvand, E. Mirzaei, M. A. Derakhshan, S. Kharrazi, E. Sadroddiny, M. Babapour, R. Faridi-Majidi, J. Appl. Polym. Sci, 2015, 132, 42133/1-42133/8.

[8] H. Y. Guan, X. H. Wang, Y. H. Guo, C. L. Shao, X. T. Zhang, Y. C. Liu, R. F. Louh, Appl. Surf. Sci., 2013, 280, 720-725.

[9] S. Y. Ryu, J. W. Chung, S. Y. Kwak, Compos. Sci. Technol., 2015, 117, 9-17.

[10] J. Liu, Z. Zhao, C. M. Xu, A. J. Duan, G. Y. Jiang, J. Rare Earth., 2010, $28,198-204$

[11] Z. M. Huang, Y. Z. Zhang, M. Kotaki, S. Ramakrishna, Compos. Sci. Technol., 2003, 63, 2223-2253.

[12] K. H. Jang, Y. O. Kang, T. S. Lee, W. H. Park, Carbohyd. Polym., 2014, $102,956-961$.

[13] Z. Y. Liu, J. J. Yan, Y. E. Miao, Y. P. Huang, T. X. Liu, Compos. Part B, 2015, 79, 217-223.

[14] S. Gao, Z. Y. Zhang, K. C. Liu, B. Dong, Appl. Catal. B, 2016, 188, 245-252.

[15] C. L. Jiang, J. Nie, G. P. Ma, RSC Adv., 2016, 6, 22996-23007.

[16] F. Li , W. M. Kang, B. W. Cheng, Y. C. Dong, Catal. Commun., 2015, 69, 150-153.

[17] B. Wang, Y. P. Wang, Y. D. Lei, N. Wu, Y. Z. Gou, C. Han, D. Fang, J. Mater. Chem. A, 2014, 2, 20873-20881.

[18] X. F. Wang, B. Ding, G. Sun, M. R. Wang, J. Y. Yu, Progr. Mater. Sci., 2013, 58, 1173-1243.

[19] H. R. Pant, H. J. Kim, M. K. Joshi, B. Pant, C. H. Park, J. I. Kim, K. S. Hui, C. S. Kim, J. Hazard. Mater., 2014, 264, 25-33.

[20] N. X. Wang, C. H. Sun, Y. Zhao, S. Y. Zhou, P. Chen, J. Lei, J. Mater. Chem., 2008, 18, 3909-3911.

[21] P. L. Zhao, L. Y. Wang, D. Liu, D. Li, P. Sun, F. M. Liu, G.Y. Lu, Electrochim. Acta, 2015, 182, 257-263. 


\title{
Graphical Abstract
}

Chin. J. Catal., 2017, 38: 73-82 doi: 10.1016/S1872-2067(16)62545-7

\section{Fabrication and catalytic behavior of hierarchically-structured nylon6 nanofiber membrane decorated with silver} nanoparticles

Huihui Zhao, Weimin Kang*, Xiaomin Ma, Nanping Deng, Zongjie Li, Bowen Cheng*

Tianjin Polytechnic University

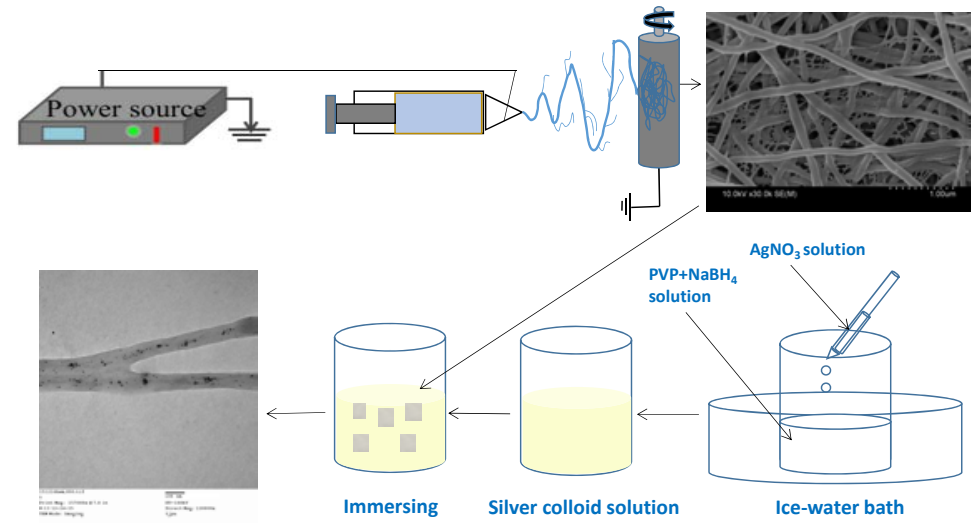

A hierarchically-structured Ag/nylon 6 (PA6) nanofiber membrane with thick fibers which acted as supports and thin fibers which provide high specific surface area was fabricated via electrospinning and impregnation method. The special structure endows the membrane high specific surface area and that contribute to the loading and dispersion of Ag NPs. Compared with normal Ag/PA6 nanofiber membrane (Ag/PA6 NM), the HS-Ag/PA6 NM with higher specific surface area exhibits improved degradation rate of methylene blue (MB) from $81.77 \%$ to $98.13 \%$ within $2 \mathrm{~h}$.

[22] M. McCune, W. Zhang, Y. L. Deng, Nano Lett., 2012, 12, 3656-3662.

[23] H. G. Wan, N. Wang, J. M. Yang, Y. S. Si, K. Chen, B. Ding, G. Sun, M. EI-Newehy, S. S. AI-Deyab, J. Y. Yu, J. Colloid Interf. Sci., 2014, 417, $18-26$.

[24] W. S. Lee, Y. S. Park, Y. K. Cho, ACS Appl. Mater. Interf., 2014, 6, 12189-12195.

[25] J. Shi, T. F. Wu, K. Y. Teng, W. Wang, M. J. Shan, Z. W. Xu, H. M. Lü, H. Deng, Mater. Lett., 2016, 166, 26-29.

[26] H. W. Bai, Z. Y. Liu, D. D. Sun, Phys. Chem. Chem. Phys., 2011, 13, 6205-6210.

[27] Z. J. Li, Y. Z. Xu, L. L. Fan, W. M. Kang, B. W. Cheng., Mater. Design, 2016, 92, 95-101.

[28] F. C. Chiu, S. M. Lai, Y. L. Chen, T. H. Lee, Polymer, 2005, 46, 11600-11609.

[29] G. M. Kim, G. H. Michler, F. Ania, F. T. B. Calleja, Polymer, 2007, 48, 4814-4823.

[30] B. Pant, H. R. Pant, D. R. Pandeya, G. Panthi, K. T. Nam, S. T. Hong, C. S. Kim, H. Y. Kim, Colloid. Surface. A, 2012, 395, 94-99.
[31] H. R. Pant, D. R. Pandeya, K. T. Nam, W. I. Baek, S. T. Hong, H. Y. Kim, J. Hazard. Mater., 2011, 189 465-471.

[32] L. Francis, F. Giunco, A. Balakrishnan, E. Marsano, Curr. Appl. Phys., 2010, 10, 1005-1008.

[33] H. Jadhav, E. Taarning, C. M. Pedersen, M. Bols, Tetrahedron Lett., 2012, 53, 983-985.

[34] K. L. Kelly, E. Coronado, L. L. Zhao, G. C. Schatz, J. Phys. Chem. B, 2003, 107, 668-677.

[35] Z. Wang, C. C. Zhao, Z. J. Pan, J. Colloid Interf. Sci., 2015, 441, 121-129.

[36] J. R. Schaefgen, C. F. Trivisonno, J. Am. Chem. Soc., 1951, 73, 4580-4585.

[37] Y. F. Zhang, M. Park, H. Y. Kim, M. EI-Newehy, K. Y. Rhee, S. J. Park, Compos. Part B, 2015, 80, 355-360.

[38] M. A. Rauf, S. S. Ashraf, J. Hazard. Mater., 2009, 166, 6-16.

[39] Y. G. Sun, Y. U. Xia, Science, 2002, 298, 2176-2179.

[40] K. Mallick, M. Witcomb, M. Scurrell, Mater. Chem. Phys., 2006, 97, 283-287.

\section{多尺度结构 Ag/PA6纳米纤维膜的制备及其催化性能}

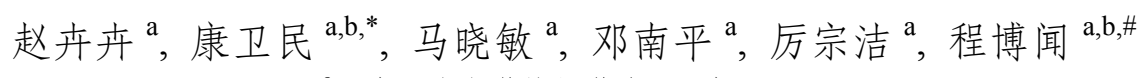 \\ 天津工业大学纺织学院, 天津 300387

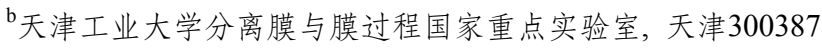

摘要: 随着工业的进步, 废水处理特别是印染废水的处理成为亟待解决的问题. 银纳米粒子因其特殊的物理化学性能而表 现出催化活性, 但银纳米粒子的团聚限制了其使用, 所以出现了一系列新的载体材料, 如微球、薄膜和纤维等. 其中电纺纳 米纤维由于具有高比表面积, 作为载体材料具有非常大的优势, 而将常规电纺纳米纤维作为载体也已有报道. 但是, 将具 有更高比表面积的电纺纳米纤维作为载体, 特别是一种类似于树枝状结构的多尺度纳米纤维作为载体还鲜有报道. 
本文制备了一种多尺度结构的 PA6 纳米纤维膜, 该纳米纤维膜由直径为 50-120 nm 的主纤维和 10-50 nm 的分支纤维 构成; 由于分支纤维的出现, 多尺度结构纳米纤维膜的比表面积得到了提高, 可以为银纳米粒子的负载提供更多附着位点. 制备的多尺度结构纳米纤维膜通过银胶溶液浸渍成功地负载银纳米粒子, 对制备的纳米纤维膜的形态、化学结构以及对亚 甲基蓝的催化性能进行了探讨.

SEM, EDS 和 TEM 结果表明, 银纳米粒子成功地负载在多尺度结构纳米纤维的表面, 并且银纳米粒子的粒径以及负载 量可以通过变换银胶溶液的浓度合理调控. 此外, 与常规 PA6 纳米纤维膜相比, 多尺度结构纳米纤维膜更有利于银纳米粒 子的分散, 同样通过银胶溶液A浸渍, 负载在多尺度结构纳米纤维上银纳米粒子粒径为 $8.6 \mathrm{~nm}$, 而负载在普通 PA6 纳米纤 维上银纳米粒子粒径为 $11.2 \mathrm{~nm}$.

XPS 分析表明, 银纳米粒子成功地负载到多尺度结构纳米纤维上, 并且经不同银胶溶液处理, 纳米纤维膜的载银量不 同. 通过 O 的高能 XPS 分析发现, 银纳米粒子与 PA6 分子间形成了配位键, 这在一定程度上有利于 Ag 纳米粒子的固定, 阻止了 $\mathrm{Ag}$ 纳米粒子的团聚.

$\mathrm{Ag} / \mathrm{PA} 6$ 纳米纤维膜以及多尺度结构 $\mathrm{Ag} / \mathrm{PA} 6$ 纳米纤维膜催化降解实验表明, 多尺度结构 $\mathrm{Ag} / \mathrm{PA} 6$ 纳米纤维膜具有较 高的催化活性, 反应 $2 \mathrm{~h}$ 后对 $10 \mathrm{mg} / \mathrm{L}$ 亚甲基蓝的降解率达到 $98.13 \%$, 并且降解过程符合伪一级动力学. 不同浸渍液浓度 处理纳米纤维膜催化实验表明, $\mathrm{Ag}$ 纳米粒子的大小以及含量都会影响纳米纤维的催化活性, 纳米粒子粒径越小, 其催化活 性越高; 不同 $\mathrm{NaBH}_{4}$ 加入量催化体系催化实验表明, 随着 $\mathrm{NaBH}_{4}$ 加入量的增大, 催化体系的降解率增高, 其对催化体系的 催化性能起着至关重要的作用; 其他条件一定, 随着染料初始浓度的增大, 催化体系的催化性能下降; 循环实验表明, 经 5 次循环之后, 其降解率仍高达 $83.5 \%$, 该纳米纤维膜具有一定的循环使用性能.

关键词: 静电纺; 多尺度结构; 银纳米粒子; PA6纳米纤维膜; 催化

收稿日期: 2015-08-05. 接受日期: 2016-08-29. 出版日期: 2017-01-05.

*通讯联系人. 电话: 13920486699 ; 电子信箱: kweimin@126.com

\#通讯联系人. 电话: 13802163663; 电子信箱: bowen15@tjpu.edu.cn

基金来源：国家自然科学基金 (51673148); 国家重点基础研究发展计划 (2014CB660813); 国家科技支撑计划 (2015BAE01B03);

中国技术创新基金(14C26211200298); 天津技术创新基金 (14TXGCCX00014, 14ZXCXGX00776).

本文的英文电子版由Elsevier出版社在ScienceDirect上出版(http://www.sciencedirect.com/science/journal/18722067). 